\title{
Vesicle propulsion in haptotaxis: A local model
}

\author{
I. Cantat ${ }^{1, a}$, C. Misbah ${ }^{2}$, and Y. Saito ${ }^{3}$ \\ ${ }^{1}$ GMCM, Université de Rennes (CNRS), Campus de Beaulieu, Bât. 11A, CS 74205 263, av. du Général Leclerc, 35042 Rennes \\ Cedex, France \\ ${ }^{2}$ Laboratoire de Spectrométrie Physique, Université Joseph Fourier (CNRS), Grenoble I, B.P. 87, 38402 Saint-Martin d'Hères \\ Cedex, France \\ ${ }^{3}$ Department of Physics, Keio University, 3-14-1 Hiyoshi, Kohoku-ku, Yokohama 223-8522, Japan
}

Received 10 February 2000

\begin{abstract}
We study theoretically vesicle locomotion due to haptotaxis. Haptotaxis is referred to motion induced by an adhesion gradient on a substrate. The problem is solved within a local approximation where a Rayleigh-type dissipation is adopted. The dynamical model is akin to the Rousse model for polymers. An invariant formulation is used to solve a dynamical model which includes a kind of dissipation due to bond breaking/restoring with the substrate. For a stationary situation where the vesicle acquires a constant drift velocity, we formulate the propulsion problem in terms of a nonlinear eigenvalue (the a priori unknown drift velocity) one of Barenblat-Zeldovitch type. A counting argument shows that the velocity belongs to a discrete set. For a relatively tense vesicle, we provide an analytical expression for the drift velocity as a function of relevant parameters. We find good agreement with the full numerical solution. Despite the oversimplification of the model it allows the identification of a relevant quantity, namely the adhesion length, which turns out to be crucial also in the nonlocal model in the presence of hydrodynamics, a situation on which we have recently reported (I. Cantat and C. Misbah, Phys. Rev. Lett. 83, 235 (1999)) and which constitutes the subject of a forthcoming extensive study.
\end{abstract}

PACS. 87.16.-b Subcellular structure and processes - 87.19.-j Properties of higher organisms - 47.55.Dz Drops and bubbles

\section{Introduction}

Phospholipidic vesicles constitute a simple model of cytoplasmic membranes of real cells. A simple model due to Helfrich [1] based on curvature energy has accounted for a variety of equilibrium shapes. The model is based on a minimal energy principle [2]. Some of the shapes (the so-called discocytes) bear strong resemblance with that of an erythrocyte (the red blood cell). Additionally, analysis of flickering (temporal small fluctuations around a given shape) of an erythrocyte by Brochard and Lennon [3] has been quite successfully described by the Helfrich model including hydrodynamics dissipation. The vesicle model has seemed then as a natural candidate, at least in a first stage, for dealing with more complex entities such as those encountered in the realm of biology. In that context, however, most of the features are of nonequilibrium dissipative nature. Very recently several theoretical [4-10] and experimental [11] investigations have been directed along that line.

We are interested here in vesicle migration, a question on which we have given recently a brief account $[6,7]$. Despite the very complex biochemical behaviour of a cell,

\footnotetext{
a e-mail: icantat@univ-rennes1.fr
}

cells may also exhibit behaviours where simple physical concepts may be evoked. It is well documented that, for example, the migration of the pronephric duct cells in salamanders is regulated by haptotaxis. Haptotaxis is a terminology that is used to express the following fact: when adhesive molecules are present in increasing amounts along an extracellular matrix (or simply on a substrate in vitro), a cell that was constantly making and breaking adhesion with such a molecule would migrate from a region of low concentration to an area where that adhesive molecule was more highly concentrated $[12,13]$. There are also evidences that cell migration during embryo development may be guided by an adhesion gradient. In other words, cell migration is here guided by a purely external physical factor, while the internal structure (the cytoskeleton) is quite unaffected on the time scale of interest. This feature drastically differs from that of a cell belonging to the immune system where the cytoskeleton plays a decisive role [14]. Despite the fact that the cytoskeleton in pure haptotaxis does not undergo a structural change as is the case during cell crawling, the problem remains very much involved since the cell cytoskeleton dissipation may come to the fore as well as an intricate bond breaking and restoring with the substrate. We shall consider here a pure vesicle moving in haptotaxis. Our belief is that advancement in this 
field can be achieved only by the progressive refinement of concepts.

We consider a vesicle moving along the substrate thanks to an adhesion gradient. As the vesicle moves, it generates hydrodynamics flow both inside and outside. Hydrodynamics induces nonlocal interactions leading to an effective coupling of two distinct regions on the vesicle. In addition, the two monolayers that form the phospholipidic membrane might slide one relative to the other. Finally, during motion the vesicle forms new bonds ahead and destroys others behind, and this process of bond breaking and restoring may be so slow that it may dominate dynamics (see later).

This paper should be regarded as using a very simplistic view in the hope of introducing the concepts of migration and to exhibit in a transparent fashion the way the problem is addressed. We shall keep the description as simple as possible. That is to say: i) we ignore nonlocality due to hydrodynamics - incorporation of hydrodynamics was briefly discussed in [7] and will be the subject of a forthcoming paper, ii) we confine ourselves to a $2 \mathrm{D}$ geometry. Some kind of dissipation due to bond breaking and restoring is introduced in our model. The adoption of a local model (no hydrodynamics) allows one to quite easily obtain analytical results and thus to extract some key ingredients about migration - especially the role of the adhesion area (length in 2D) - which turns out to be crucial also when hydrodynamics is included.

The scheme of this paper is organized as follows. In Section 2 we write down the equations of motion and comment them. In Section 3 we present a forward time integration and present the main result. Section 4 presents the solution of the stationary system in a form of a nonlinear eigenvalue problem, where the drift velocity is the eigenvalue. In Section 5 we give an analytical solution. A conclusion and a discussion are presented in Section 6 .

\section{Equation of motion}

\subsection{Parameterization}

We consider an adhering vesicle, deposited on a flat substratum which is oriented by its normal vector $\hat{\mathbf{y}}$ (Fig. 1 ). The $x$-axis is along the wall and represents the direction of vesicle motion occurring by convention from left to right. As stated before we confine ourselves to two dimensions. That is to say, the vesicle morphology is invariant in the $z$-direction, similar to a tubular vesicle.

The interaction between the vesicle and the substrate is taken into account by introducing an adhesion potential. The range of the potential in realistic situations (typically several $\mathrm{nm}$ ) is small in comparison to the vesicle size (several $\mu \mathrm{m})$, so that it is justified in practice to consider a contact potential, unless otherwise indicated (see later). The energy interaction is then zero if $y>0$ and is nonvanishing only close to contact (if $y=0$ ). At the junction point between the free part of the vesicle (whose length is denoted as $L^{*}$ ) and the adhered part (with length $L_{\mathrm{adh}}$ ), the potential undergoes an abrupt change. The contact

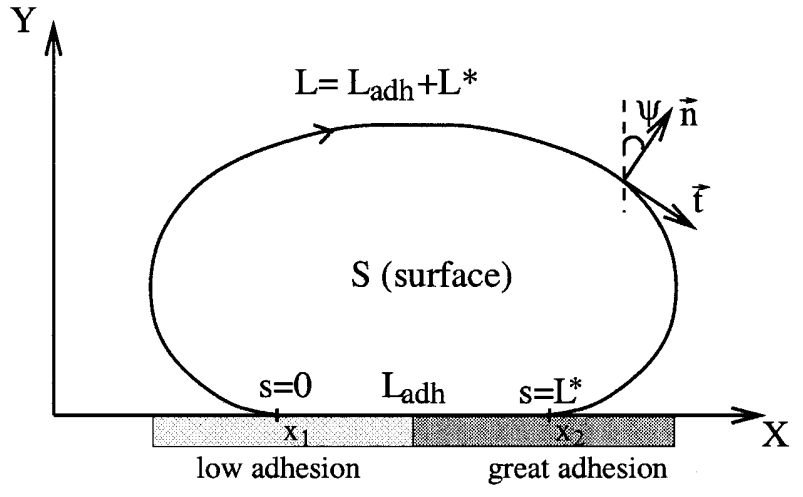

Fig. 1. Notations used in the text.

between the membrane and substrate occurs at two welldefined points $x_{1}$ on the left and $x_{2}$ on the right. These parameters are related to the total length of the curve $L$ by $L=L_{\text {adh }}+L^{*}=\left(x_{2}-x_{1}\right)+L^{*}$. We use an intrinsic representation of the vesicle contour by introducing $\psi(s)$, the angle between the outward normal and the $y$-axis, and $s$ the arclength, as shown in Figure 1. We only need to consider the function $\psi(s, t)$ from $s=0$ to $s=L^{*}$ corresponding to the contact points $x_{1}$ and $x_{2}$, respectively. Because of the contact potential character the adhesion length is completely fixed if the two contact points $x_{1}$ and $x_{2}$ are known. Thus the vesicle shape and its dynamical properties (like the propulsion velocity) are known if $x_{1}, x_{2}$ and the function $\psi(s, t)$ are determined. The demand that the parametrization of the vesicle be compatible with the adhesion on the substrate is fulfilled by the two geometrical constraints : i) the distance between both contact points, $x_{2}-x_{1}$, must coincide with the adhesion length $L_{\text {adh }}=x_{2}-x_{1}$, ii) their vertical coordinates $y_{1}$ and $y_{2}$ must have the same value, $y_{2}-y_{1}=0$. These two constraints can be expressed in terms of $\psi(s, t)$. For that purpose we use the relations

$$
\frac{\partial x}{\partial s}=\cos \psi, \quad \frac{\partial y}{\partial s}=-\sin \psi
$$

which allow us to write the two constraints in the following form:

$$
\begin{aligned}
& \int_{0}^{L^{*}} \frac{\partial x}{\partial s} \mathrm{~d} s=\int_{0}^{L^{*}} \cos \psi(s) \mathrm{d} s=x_{2}-x_{1}=L_{\mathrm{adh}} \\
& \int_{0}^{L^{*}} \frac{\partial y}{\partial s} \mathrm{~d} s=\int_{0}^{L *}-\sin \psi(s) \mathrm{d} s=y_{2}-y_{1}=0
\end{aligned}
$$

These are the geometrical constraints. In order to describe vesicle dynamics, we need a dynamical equation for the evolution of $\psi(s, t)$. A movement of the vesicle (due, for example, to an adhesion gradient) is limited by dissipation (such as hydrodynamics etc...). The vesicle reacts to any deviation from equilibrium by its internal forces (bending, possible stretching —or resistance to stretching). Let us first discuss these forces. 


\subsection{Energy and forces}

All the relevant membrane properties are summarized in the following energy, expressed in $2 \mathrm{D}$, with the dimension of an energy per unit length:

$$
E=\int_{\mathcal{C}} \kappa \frac{\left(c-c_{\mathrm{s}}\right)^{2}}{2} \mathrm{~d} s-\int_{x_{1}}^{x_{2}} w(x) \mathrm{d} x+\int_{\mathcal{C}} \zeta(s) \mathrm{d} s+p S .
$$

The first term is the well-known Helfrich curvature energy, with the rigidity $\kappa$, the curvature $c=\partial \psi / \partial s$ and the spontaneous curvature $c_{s}[1]$. Because of the 2D-specific conservation law $\int c \mathrm{~d} s=2 \pi$, any curve displacement leaves unchanged the energy terms associated to the spontaneous curvature. We can thus omit the term associated with $c_{\mathrm{s}}$. The second term expresses the adhesion energy and is only integrated on the adhered part of the curve. As we are concerned with an inhomogeneous substratum, the contact potential depends on the variable $x$ and is denoted by $-w(x)$ (with $w>0$, meaning that adhesion is favorable). Finally, the last two terms ensure the length and surface conservation, respectively. The membrane is a two-dimensional incompressible fluid. The phospholipid exchange with the solvent is virtually absent, and the area per molecule on the vesicle remains constant. This leads to the local length conservation (in the 2D language). The variable $\zeta$ is a local Lagrange multiplier which enforces the arclength $\mathrm{d} s$ to a constant value. The enclosed surface $S$ conservation is a consequence of the membrane impermeability and fluid incompressibility. It is ensured by the global Lagrange multiplier $p$. The interpretation of $\zeta$ and $p$ as a tension and a pressure will be discussed later.

The functional derivative of the energy (4) provides us with the various forces acting on the membrane.

\subsubsection{Curvature forces}

Under the assumption that the membrane is completely flat on the adhered part, we reduce the integration domain of the first energetic term in equation (4) to $\left[0, L^{*}\right]$. Making use of the relations $\mathbf{t}=\mathbf{r}^{\prime}$ and $\mathbf{n}=-(1 / c) \mathbf{r}^{\prime \prime}$ (the prime designates derivative with respect to $s$ ) which are the membrane tangential and normal unit vectors, we obtain for the curvature energy $E_{\mathrm{c}}$

$$
E_{\mathrm{c}}=\frac{\kappa}{2} \int_{0}^{L^{*}}\left(\frac{\partial^{2} \mathbf{r}}{\partial s^{2}}\right)^{2} \mathrm{~d} s .
$$

When taking the functional derivative of $E_{\mathrm{c}}$ with respect to the position, care must be taken. Indeed the arclength element must also undergo a variation. A convenient formulation avoiding confusion rests on the introduction of a general parametrization $a \in[0,1]$, related to $s$ by the metric $g=(\mathrm{d} s / \mathrm{d} a)^{2}$. The energy expression becomes $[8,15]$

$$
E_{\mathrm{c}}=\frac{\kappa}{2} \int_{0}^{1}\left[\left(\frac{\partial^{2} \mathbf{r}}{\partial a^{2}}\right)^{2}-\frac{1}{g}\left(\frac{\partial^{2} \mathbf{r}}{\partial a^{2}} \frac{\partial \mathbf{r}}{\partial a}\right)^{2}\right] g^{-3 / 2} \mathrm{~d} a .
$$

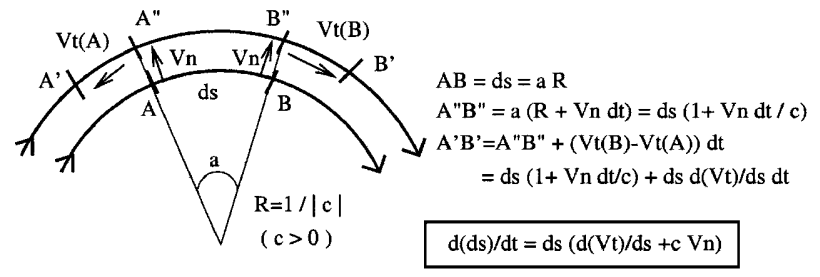

Fig. 2. A geometrical explanation of the arclength variation with time.

The functional derivative, though straightforward, may be too lengthy if one does not take care in regrouping adequately various terms as explained in [8]. The result can be written in a simple form:

$$
\mathbf{f}_{\mathrm{c}}=-\frac{\delta E_{\mathrm{c}}}{\delta \mathbf{r}(s)}=\kappa\left(\frac{\partial^{2} c}{\partial s^{2}}+\frac{c^{3}}{2}\right) \mathbf{n} .
$$

The curvature force is, as expected, free of any tangential contribution. The first term in equation (7), involving the second derivative of the curvature, tends to keep curvature repartition as homogeneous as possible. It is also present in 3D under the more complicated form of the Laplace-Beltrami operator. The second term proportional to $c^{3}$ is in contrast $2 \mathrm{D}$-specific. It tends to increase the size of any convex shape. Note that in $3 \mathrm{D}$ the curvature energy is scale invariant, which implies a vanishing curvature force of this type on a sphere. The difference between $2 \mathrm{D}$ and $3 \mathrm{D}$ can be explained in the following way. Let us consider a finite cylinder of length $H$ and radius $R \ll H$, which constitutes a good approximation for an infinitely long cylinder. In order to make the cylinder "closer" to a sphere, which is the corresponding 3D equilibrium shape, the curvature force would tend to increase the radius and decrease the length so as to bring the cylinder shape as close as possible to a sphere. This gives an intuitive picture of the $c^{3}$ term in $2 \mathrm{D}$.

In the discussion above we did omit the boundary contribution when taking the functional derivative. Since this point is a bit subtle we have postponed it to the end of this section.

\subsubsection{Length and surface constraints}

On the free part of the curve, the force which is associated to the first Lagrange multiplier $\zeta$ is obtained upon functional derivation of $E_{1}=\int \zeta \mathrm{d} s$. The result is

$$
\mathbf{f}_{\mathrm{l}}=-\delta E_{\mathrm{l}} / \delta \mathbf{r}(s)=-c \zeta(s) \mathbf{n}+\frac{\mathrm{d} \zeta}{\mathrm{d} s} \mathbf{t} .
$$

The normal component is easily identified as a Laplace pressure, whereas the tangential one looks like a Marangoni force (which is encountered when surface tension is inhomogeneous). However, $\zeta$ is not exactly similar to a surface tension as for an interface between two fluids. The "tension" $\zeta$ is not an intrinsic property of the membrane. It adapts itself to the other forces in order to 
maintain the local length fixed. In other words, the problem is implicitly written in a thermodynamical ensemble with fixed length. This differs from the usual problem for fluid or solid surfaces where the surface tension is fixed instead. Thus $\zeta$ is a variable that must be determined self-consistently as a Lagrange multiplier, by use of the constraint equation (see appendix in Ref. [16]):

$$
0=\frac{\partial(\mathrm{d} s)}{\partial t}=\left(\frac{\partial v_{\mathrm{t}}}{\partial s}+c v_{\mathrm{n}}\right) \mathrm{d} s
$$

This relation (9) simply expresses the condition of vanishing velocity divergence on the curved contour of the vesicle, which is precisely the incompressibility condition in the $2 \mathrm{D}$ fluid constituting the membrane (written here in one dimension). A more intuitive way of viewing expression (9) is presented in Figure 2. The Marangoni term is the only tangential term among all membrane forces (see Eqs. $(7,8,10))$. It is seen from (8) that the Lagrange multiplier must be uniform at equilibrium. For the sake of simplicity and in order to get more insight into analytical understanding, a uniform value will be assigned to $\zeta$, even out of equilibrium. A discussion of this point will be presented in Section 6. This assumption implies some consequences on dynamics (and especially on the tangential velocity) which will be presented in Section 2.3.

Finally, we have to consider the force associated to $E_{\mathrm{s}}=p S$ :

$$
\mathbf{f}_{\mathrm{s}}=-\delta E_{\mathrm{s}} / \delta \mathbf{r}(s)=-p \mathbf{n}
$$

The Lagrange multiplier $p$ depends only on time; it enforces a constant area. Two physical interpretations can be invoked depending on the situation under consideration. Either we consider an impermeable membrane, and $p$ would be the hydrostatic pressure difference between outside and inside; or we choose a model of permeable membrane and $p$ would play the role of an osmotic pressure. Both models are equivalent as long as we do not consider hydrodynamic flows.

\subsubsection{Adhesion forces and boundary terms}

The functional derivative induces boundary terms at each contact point. The additional variation $\delta E_{\mathrm{c}}^{\mathrm{b}}$ and $\delta E_{\mathrm{w}, \mathrm{l}}^{\mathrm{b}}$ for the curvature, adhesion and tension energies, associated with a small displacement $\delta \mathbf{r}$ of the contact points is given by (see $[8,15])$

$$
\begin{aligned}
& \delta E_{\mathrm{c}}^{\mathrm{b}}=\left[\delta \dot{\mathbf{r}} \cdot\left(-\frac{\kappa c}{\sqrt{g}} \mathbf{n}\right)\right]_{0}^{L^{*}}+\left[\delta \mathbf{r} \cdot\left(\kappa \frac{\partial c}{\partial s} \mathbf{n}-\frac{\kappa c^{2}}{2} \mathbf{t}\right)\right]_{0}^{L^{*}} \\
& \delta E_{\mathrm{w}, 1}^{\mathrm{b}}=[\delta \mathbf{r} \cdot(\zeta \mathbf{t}+(\zeta-w(x)) \hat{\mathbf{x}})]_{0}^{L^{*}} .
\end{aligned}
$$

Following the definition of these boundary points, they remain on the substrate. Thus, the accessible values for $\delta \mathbf{r}$ is then reduced to $\delta \mathbf{r} \propto \hat{\mathbf{x}}$. Additionally, in order to keep the curvature energy finite, we impose a vanishing value for

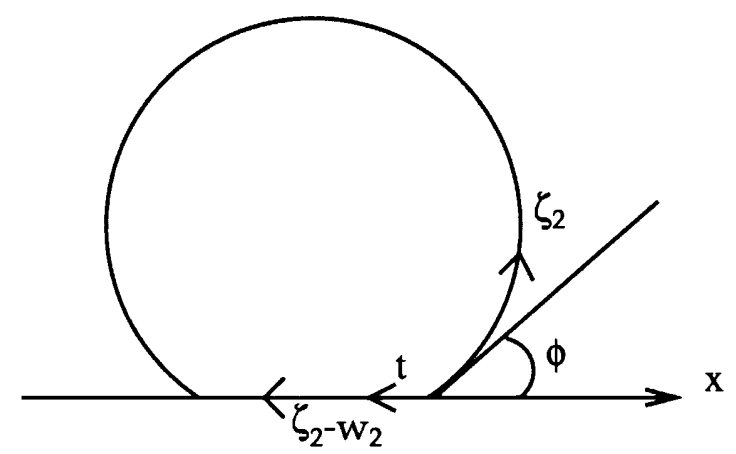

Fig. 3. Force equilibrium at the fore contact point in the small rigidity limit.

the contact angle $\phi$ between the membrane and the substrate (see Fig. 3). Within our formulation, this constraint does not follow from the energy minimization and has thus to be added into the physical model. More precisely, at the discontinuity point (say $x_{2}$ ) one has to add to the Helfrich energy a term of the form $\kappa(\Delta \psi / \Delta s)^{2}$ which informs us on how would the vesicle on the adhered part feel, so to speak, the behavior of the vesicle at the junction point on the right side. Across the contact point of a vanishing extent, $\Delta s \rightarrow 0$, while the angle, if it had to have another value than zero, would make a jump leading to an abnormally increasing curvature energy. We must then impose a vanishing contact angle. These various conditions (motion along the wall and a vanishing contact angle) lead to $\mathbf{n}=-\hat{\mathbf{y}}, \mathbf{t}=-\hat{\mathbf{x}}$ and $\delta \dot{\mathbf{r}} \propto \hat{\mathbf{x}}$. It follows then that the term proportional to $\delta \dot{\mathbf{r}}$ in equation (11) vanishes automatically. The second term becomes $\kappa / 2\left(c_{2}^{2} \delta x_{2}-c_{1}^{2} \delta x_{1}\right)$ with $c_{1}$ and $c_{2}$ the curvatures on the left and right contact points. These terms are counterbalanced by adhesion and tension terms (eq. (12)) leading to the relation

$$
\frac{\delta E}{\delta x_{i}}=\mp\left(\kappa \frac{c_{i}^{2}}{2}-w\left(x_{i}\right)\right) \hat{\mathbf{x}},
$$

where the - and + signs refer to the rear and fore contact points represented by the subscript $i=1,2$. At equilibrium, we recover here the relation $c=\sqrt{2 w / \kappa}$ [17].

The energy variation given by equation (13) cannot really be identified as a physical force. It corresponds indeed to a geometrical point displacement. The "force" orientation is here parallel to the substrate, whereas the real force acting on the contact point, considered as a material point, is expected to be normal to the substrate. As we have seen above, the curvature forces are indeed normal when applied to a an adjacent piece of the membrane (see eq. (7)). The present "force" has the meaning of how much energy would be involved in displacing the contact point from one position to another. That geometrical point is by its very nature sitting on the substrate, so that the "force" associated with its displacement is naturally tangential.

We find it worthwhile to make a short digression. Suppose that the angle is not fixed to zero as we did above. More precisely, suppose that the rigidity is so small or the adhesion is so large (see below what this does mean) 
then the vesicle will be so tense that it would look like a droplet outside some length scale $\ell$ to be determined below (of course, within that scale, which is sufficiently close to the substrate, the matching must be tangential). If we do not assume a value for the angle (that is no relation between $\mathbf{n}$ or $\mathbf{t}$ with $\hat{\mathbf{x}}$ and $\hat{\mathbf{y}}$ ), and set $\delta \mathbf{r} \propto \hat{\mathbf{x}}$ we find from (13) that $c=0$ at the contact (which means a straight line at the contact) and that the angle between that line and the substrate obeys

$$
\begin{aligned}
w_{i} & =\left(1-\cos \left(\phi_{i}\right)\right) \zeta \\
\phi_{i} & \sim \sqrt{\frac{2 w_{i}}{\zeta}} \text { (for small angles) }
\end{aligned}
$$

which is nothing but the Young condition $[2,18]$. We have neglected $\kappa \partial c / \partial s$ in comparison to $w$. The justification is as follows. $\kappa \partial c / \partial s \sim \kappa c_{0} / \ell$, where $c_{0}$ is the true contact curvature given by $\sqrt{2 w / \kappa}$. The approximation is legitimate provided that the length scale $\ell \gg \sqrt{\kappa / w}$. The length $\sqrt{\kappa / w}$ is the radius of curvature at contact. If the scale of interest is outside that internal region, then the droplet limit is justified. It must be emphasized however that the effective contact angle is not an intrinsic property of the adhered membrane, as for a droplet, but it is linked to other parameters (rigidity, the vesicle scale - on which $\zeta$ depends - etc...). In particular, the tension $\zeta$ is fixed by the reduced volume, which is a global property of the vesicle: different vesicles of the same phospholipid composition, but with different sizes, may have different contact angle on the same substrate.

\subsection{Dynamical equation}

An important point which must be emphazised when dealing with dynamics is the identification of the dissipation sources. These are the following: i) the dissipation in the membrane via molecule rotations (very much like liquid crystals where dissipation is characterized by the Leslie coefficient); ii) hydrodynamics flows inside and outside the vesicle; iii) friction between the monolayers; and iv) bond breaking and restoring with the substrate. It is well known that dissipation associated with rotation (internal dissipation) is negligible in practice [3], and for free vesicles (no substrate) hydrodynamics seems to be the most important dissipation. Hydrodynamics induces nonlocal interactions [7] and this will be dealt with extensively in a forthcoming paper. Our wish in this paper is to present a pedestrian model, namely a local one, which allows for a complete analytical solution that will help to identify some key ingredients in the migration process. A specific dissipation with the substrate will be introduced later. For the moment we confine our description to the free vesicle case. The local model to be presented here is similar to the so-called Rousse model [19] in the community of polymers. Indeed, for a one-dimensional contour in a three-dimensional space dynamics becomes local even in the presence of hydrodynamics [20].
Within a simplistic picture of local dynamics, the natural choice for the dissipation function is

$$
F_{\mathrm{d}}=\frac{\eta}{2} \int|\mathbf{v}|^{2} \mathrm{~d} s
$$

The coefficient $\eta$ is here an effective viscosity and has the dimension of a viscosity per unit length. Its numerical value is estimated by $\eta=\eta_{\text {wat }} / R \sim 10^{2} \mathrm{kgm}^{-2} \mathrm{~s}^{-1}$, with $\eta_{\text {wat }}$ the water viscosity and $R$ a typical vesicle size.

Neglecting inertial terms, the Euler-Lagrange equations become then

$$
-\frac{\delta E}{\delta \mathbf{r}}=\frac{\delta F_{d}}{\delta \mathbf{v}} \Rightarrow \eta \mathbf{v}=\mathbf{f} .
$$

As expected, we find a local proportionality between the membrane velocity $\mathbf{v}$ and the membrane force $\mathbf{f}$, which is a nonlinear function of position. In the present picture where the effective tension $\zeta$ is space independent no tangential force appears so that physics will only fix the normal velocity, while the tangential velocity has no physical meaning as described below.

\subsubsection{Normal velocity}

The normal membrane force is (see Eqs. $(7,8,10)$ ):

$$
f_{\mathrm{n}}=\kappa\left(\frac{\partial^{2} c}{\partial s^{2}}+\frac{c^{3}}{2}\right)-c \zeta-p
$$

From the dynamical law (16) and the membrane forces expression (17) we obtain the normal velocity as a function of $\psi(s)$ :

$$
v_{\mathrm{n}}(s)=f_{\mathrm{n}}=\frac{\kappa}{\eta}\left[\frac{\partial^{3} \psi}{\partial s^{3}}+\frac{1}{2}\left(\frac{\partial \psi}{\partial s}\right)^{3}-\frac{\zeta}{\kappa} \frac{\partial \psi}{\partial s}-\frac{p}{\kappa}\right]
$$

It is convenient to write the dynamical equation in terms of the angle $\psi$ and not the curvature $c$. The reason is that the boundary conditions are written naturally as a function of $\psi$ (tangential matching, $\psi= \pm \pi$, and contact curvature $\partial \psi / \partial s=\sqrt{2 w / \kappa})$.

\subsubsection{Tangential velocity}

There is only one tangential contribution to the membrane forces, $\partial \zeta / \partial s$, which is zero with the assumption of a uniform tension (see Eq. (8)). This implies that only the total length is conserved, and not the local one. A dilatation of a part of the membrane is then permitted, as long as the remaining part of the vesicle is contracted in order to keep the total length unchanged (see Fig. 4). Within this approximation, there is no energy variation associated to tangential motion, and therefore no forces. In other words, we consider the vesicle contour as a mathematical curve, loosing the concept of density: only the shape matters, independently of the points distribution on the curve. 


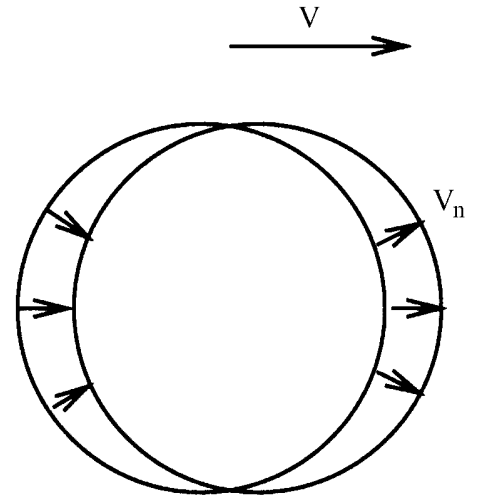

Fig. 4. Translation of a circle obtained with a purely normal motion. The right part is dilated, whereas the left part is contracted.

We could equivalently assume that only the normal velocity contributes to dissipation. In that case, the dissipation function (15) would take the form $F_{\mathrm{dn}}=$ $\eta / 2 \int\left|v_{\mathrm{n}}\right|^{2} \mathrm{~d} s$ and the equation of motion (16) becomes $\mathbf{f}=\eta v_{\mathrm{n}} \mathbf{n}$. The tangential force $\partial \zeta / \partial s$ must then vanish and we get automatically that $\zeta=$ constant. Thus our assumption of a global Lagrange multiplier can also be viewed as the result of a dissipation due uniquely to normal displacements.

As we have already mentioned, tangential displacements do not induce a geometrical change. We are thus at liberty to choose one which is convenient, without physical meaning, interpreted as a reparametrization of the curve.

In the present model the most convenient parametrization requires a homogeneous points distribution along the free part of the curve, which is expressed as $\mathrm{d} / \mathrm{d} t\left(s(a) / L^{*}\right)=0$. This provides the expression for the "nonphysical tangential velocity" that can be seen as a gauge, without influence on the motion (see appendix in Ref. [16]):

$$
\begin{aligned}
v_{\mathrm{t}}(s)= & v_{\mathrm{t}}(0)-\int_{0}^{s} c v_{\mathrm{n}} \mathrm{d} s \\
& +\frac{s}{L^{*}}\left(\int_{0}^{L^{*}} c v_{\mathrm{n}} \mathrm{d} s+v_{\mathrm{t}}\left(L^{*}\right)-v_{\mathrm{t}}(0)\right) .
\end{aligned}
$$

If the free length $L^{*}$ remains constant during the motion, as happens for a stationary regime, equation (19) imposes nothing but a constant distance between two consecutive points on the vesicle. The local length conservation (9), which is physical, seems then surprisingly to be fullfilled. In reality, once we have adopted a contant tension - implying a vanishing tangential physical velocity - any point distribution is of purely geometrical nature, and we could impose another gauge than the above one, without affecting the physics. On the other hand, had we considered $\zeta$ to be nonconstant, we would then have obtained a physical tangential velocity, which would act on the normal one through the Lagrange multiplier. Indeed, use of equation (9) fixes $\zeta(s)$ which in turn acts on the value of $v_{\mathrm{n}}$, and then on physics. In the simplistic model we adopt, the tangential velocity is determined a posteriori, independently of the normal velocity. That is why it is only a nonphysical reparametrization, a "gauge". A remark is in order: in this situation the question of a rolling or sliding motion does not make sense, since both motions differ only by a tangential velocity.

The membrane velocity is given as a function of $\psi(a)$, $a$ being the auxiliary parametrization of the free part of the vesicle, running from one contact point to the other. In order to obtain a closed system we need a relation between the evolution of $\psi(a)$ and the velocities. The temporal derivative of $\psi$, for a given $a$, is presented in the appendix of reference [16]:

$$
\left.\frac{\partial \psi}{\partial t}\right)_{a}=c v_{\mathrm{t}}-\frac{\partial v_{\mathrm{n}}}{\partial s} .
$$

The last step is the determination of the boundary conditions at the contact with the substrate.

\subsubsection{Contact points velocity}

The motion of the contact point is governed by a binding/unbinding mechanism, implying a dissipation law that differs from the bulk dissipation. The most natural way for introducing a dissipation law is the following (with $\Gamma$ a phenomenological dissipation coefficient):

$$
\Gamma \frac{\mathrm{d} x_{i}}{\mathrm{~d} t}=-\frac{\delta E}{\delta x_{i}} .
$$

Using the energy variation (13) we get the following dynamical law, with $w_{i}=w\left(x_{i}\right)$ and $v_{i}=\mathrm{d} x_{i} / \mathrm{d} t$,

$$
c_{1}=\sqrt{\frac{2 w_{1}+\Gamma v_{1}}{\kappa}}, \quad c_{2}=\sqrt{\frac{2 w_{2}-\Gamma v_{2}}{\kappa}},
$$

which constitute the dynamical boundary conditions. These out-of-equilibrium values for the curvature are quite intuitive: the unbinding delay at the rear point induces a larger curvature than at equilibrium, whereas the binding delay at the fore point induces a smaller curvature.

\section{Transient behavior}

The formalism presented in the first part lends itself very well to analytical computation and stationary shape determination, as will appear in the following paragraphs. Nevertheless, having access to the transient process is highly desirable. In particular, it checks the dynamical stability of an eventual stationary behavior, obtained after a relaxation. The successive vesicle profiles are determined by a direct numerical implementation of the dynamical equations (18), (19) and (20). Unfortunately, numerical instabilities are difficult to avoid around each contact point (due to a contact adhesion potential). A smoother model, without discontinuities, is more convenient for such an approach. For this reason, in this paragraph devoted to transient processes, the adhesion potential will be supposed to 
be of small, but nonvanishing, range. We rapidly summarize below the small technical changes arising from this model modification. The chosen potential profile is

$$
w(\mathbf{r})=w_{0}\left(1+u_{0} x\right)\left(\frac{y_{0}^{4}}{y^{4}}-\frac{2 y_{0}^{2}}{y^{2}}\right),
$$

with the new length $y_{0}$ fixing the characteristic distance between the substrate and the membrane, and $\hat{w}(x)=$ $-w_{0}\left(1+u_{0} x\right)$ the minimum of the potential interaction, occurring for $y=y_{0}$. It plays the role of the previous $w(x)$. It depends linearly on $x$ with an adhesion gradient $u_{0}$. The distance $y_{0}$ is chosen of the order of $10 \mathrm{~nm}$, which is small enough with respect to the vesicle size to introduce only small variations between both models. In this case the parametrization is performed on the whole closed curve, and the boundary terms (Eqs. (22)) are no more relevant. The adhesion forces $\mathbf{f}_{w}$ are obtained by functional derivation of $\int w(\mathbf{r}) \mathrm{d} s$ leading to

$$
\mathbf{f}_{w}=-(c w+\nabla w \cdot \mathbf{n}) \mathbf{n} .
$$

Additionally, the gauge condition fixing the tangential velocity equation (19) is simplified: the velocity $v_{\mathrm{t}}(0)$ is supposed to be zero, without loss of generality, so the first term disappears; the last term is proportional to the length variation of the total parametrized curve, which is zero because we consider the complete profile and no more the free part of the curve. Thus we obtain for the gauge, replacing equation (19)

$$
v_{\mathrm{t}}=-\int_{0}^{s} \mathrm{~d} s v_{\mathrm{n}} c
$$

Using equations (24) and (25) we finally get the dynamical equation for $\mathbf{r}$ :

$$
\begin{aligned}
\frac{\partial \mathbf{r}(a, t)}{\partial t}= & {\left[\kappa\left(\frac{\partial^{2} c}{\partial s^{2}}+\frac{1}{2} c^{3}\right)-c w\right.} \\
& -(\nabla w \cdot \mathbf{n})-p-\zeta c] \mathbf{n}+v_{\mathrm{t}} \mathbf{t} .
\end{aligned}
$$

The Lagrange multipliers are determined from the following constraint equations:

$$
\begin{aligned}
& \frac{\mathrm{d} L}{\mathrm{~d} t}=\int c v_{\mathrm{n}} \mathrm{d} s=0, \\
& \frac{\mathrm{d} S}{\mathrm{~d} t}=\int v_{\mathrm{n}} \mathrm{d} s=0 .
\end{aligned}
$$

The normal component of the velocity in equation (26) will be denoted by convention as $v_{\mathrm{n}}=v_{\mathrm{n}}^{0}-p-c \zeta$. With this notation, equations $(27,28)$ appear as a very simple linear equation system in $\zeta$ and $p$. Its solution provides the pressure and tension values:

$$
\begin{gathered}
\zeta=\frac{\left\langle c v_{\mathrm{n}}^{0}\right\rangle-\langle c\rangle\left\langle v_{\mathrm{n}}^{0}\right\rangle}{\left\langle c^{2}\right\rangle-\langle c\rangle^{2}}, \\
p=-\zeta\langle c\rangle+\left\langle v_{\mathrm{n}}^{0}\right\rangle .
\end{gathered}
$$

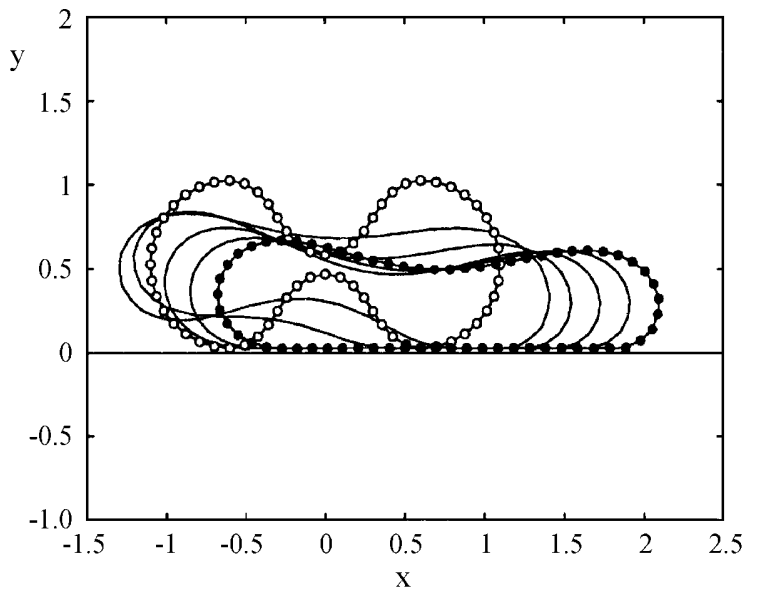

Fig. 5. Successive vesicle profiles. The first one with open circles is an arbitrarily chosen initial shape. It relaxes to a permanent shape marked by filled circles on an inhomogeneous substrate.

with the average defined by

$$
\langle\cdots\rangle \equiv \frac{1}{L} \int_{0}^{L} \mathrm{~d} s \cdots
$$

We are now in a position to deal with the numerical anlysis. The dynamics is overdamped and for this reason local in time. Starting from an arbitrary profile, forward time integration provides us with the vesicle evolution. We have checked that i) for a free vesicle (no substrate) the shape (with no external force) tends towards that obtained by direct energy minimization; ii) we have also checked that for a homogeneous substrate an arbitrary initial shape evolves after some time to the shapes obtained in [17] by direct energy minimization.

Let us now turn to the nonequilibrium situation ensured by an adhesion gradient. Starting from an initial shape, the vesicle acquires a nonsymmetric shape and moves in the gradient direction. After transients have decayed the vesicle acquires a permanent regime with a constant velocity. Figure 5 shows the shape evolution.

\section{Stationary motion: direct numerical solution}

The formulation of our problem in terms of a direct stationary problem is very convenient for a systematic study of the velocity evolution as a function of various parameters. It will also allow us to present a simple analytical solution. It is convenient here to come back to the contact potential model. For a vesicle which has attained a stationary shape and velocity $V$ the equations become steady with $V$ as an unknown parameter.

For a stationary motion along the $x$-axis, normal and tangential velocities can be written as functions of the angle $\psi$ and of the translational velocity $V$ :

$$
\begin{aligned}
& v_{\mathrm{n}}=V \hat{\mathbf{x}} \cdot \hat{\mathbf{n}}=V \sin \psi, \\
& v_{\mathrm{t}}=V \hat{\mathbf{x}} \cdot \hat{\mathbf{t}}=V \cos \psi .
\end{aligned}
$$




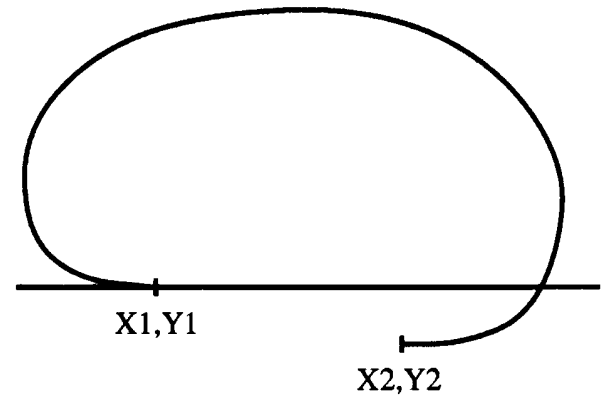

Fig. 6. Geometrical constraint on the curve.

The shape and velocity are entirely determined from the relation between normal velocities and forces. The equation of motion is obtained from equations $(18,32)$ :

$$
V \sin \psi=\frac{\kappa}{\eta}\left[\frac{\partial^{3} \psi}{\partial s^{3}}+\frac{1}{2}\left(\frac{\partial \psi}{\partial s}\right)^{3}-\frac{\zeta}{\kappa} \frac{\partial \psi}{\partial s}-\frac{p}{\kappa}\right] .
$$

Let us present briefly a counting argument showing that the problem is well defined. Equation (34) is a nonlinear third-order differential equation for $\psi$, with 3 parameters to be determined: $\zeta, p$ and $V$. So we need 6 "pieces of information".

We have the following equations at our disposal:

- 2 geometrical constraints (eqs. (2) and (3)):

$$
\int_{0}^{L^{*}} \cos \psi(s) \mathrm{d} s=L_{\mathrm{adh}}, \int_{0}^{L *}-\sin \psi(s) \mathrm{d} s=0
$$

- 4 boundary equations corresponding to the contact angles and their first derivatives (the dynamical contact curvatures $c_{1}$ and $c_{2}$ given by equation (22))

$$
\begin{aligned}
& \psi_{1} \equiv \psi(s=0)=-\pi, \quad \psi_{2} \equiv \psi\left(s=L^{*}\right)=\pi \\
& \left.\left.\frac{\partial \psi}{\partial s}\right)_{s=0}=c_{1}, \quad \frac{\partial \psi}{\partial s}\right)_{s=L^{*}}=c_{2}
\end{aligned}
$$

- 1 equation ensuring that the enclosed surface is equal to the prescribed area;

- 1 equation ensuring that the total length of the curve is precisely the prescribed one, $L$, which is related to the two other lengths by

$$
L=L^{*}+L_{\text {adh }} .
$$

There are thus 8 conditions, for only 6 pieces of information needed. The system seems then to be overdetermined. This is not the case. Indeed it must be noted that the problem involves additional unknowns which are $L^{*}$ and $L_{\text {adh }}$. So in reality we have 8 unknown parameters as well. The problem is thus well defined.

Once the shape is determined we must in principle evaluate the area and change the parameter $p$ until the area coincides with the prescribed one. But since the area is a conjugate variable to $p$ we can fix $p$-which is more

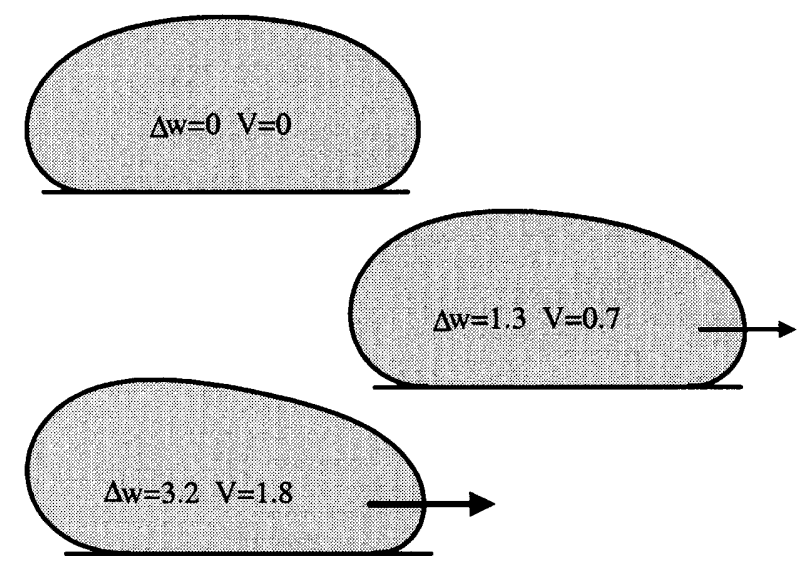

Fig. 7. Out of equilibrium adhering vesicle profiles. $V$ is measured in units of $100 \mu \mathrm{m} / \mathrm{s}$ and $W$ in units of $10^{-4} \mathrm{~mJ} / \mathrm{m}^{2}$.

convenient - and this will fix some area that is treated as free (not imposed in advance). Additionally, we are at liberty to prescribe $L$ (that fixes some length scale). $L_{\text {adh }}$ can then be determined if $L^{*}$ is known; $L_{\text {adh }}$ can thus be removed from the problem upon using equation (38). The first constraint (35) becomes then

$$
\int_{0}^{L^{*}} \cos \psi(s) \mathrm{d} s=L-L^{*} .
$$

In other words, prescribing the total length to $L$ and the pressure to $p$ lowers the number of unknowns by two. This is so because we do not want to have a specific area, and that $L^{*}$ and $L_{\text {adh }}$ are not independent if we treat the total length as known. That is to say, we have finally 6 fixed boundary conditions or constraints (35-37) and six parameters which are $L^{*}, V$ and $\zeta$, plus three constants of integration due to the third-order differential equation (34).

Once the problem is solved the vesicle shape is obtained by making use of equations (1).

If $w_{1}=w_{2}$, the vesicle is at equilibrium on a homogeneous substrate and one obviously expects a vanishing velocity. This comes out automatically from the above formulation. If we were interested from the beginning in an equilibrium problem, we would then not have introduced $V$ as an unknown parameter. In that case because the profile is symmetric the second condition (35) is automatically satisfied.

When $w_{1} \neq w_{2}$, there is no equilibrium solution for the vesicle, which has to move towards the stronger adhesion region. If we impose a vanishing velocity there is no way to fulfill the second condition (35) (a typical profile would be the one shown in Fig. 6) where starting from one end we arrive at the other end at a different height. Arriving at the same height can be achieved only for a specific velocity (or at most a discrete set of solutions), the one we are seeking. Thus the second condition of equation (35) (which is parametrized by the set of $P_{i}$ ) can be viewed as "quantization" condition. This is a nonlinear eigenvalue problem of Barenblat-Zeldovitch type.

The numerical solution reveals an out-of-equilibrium shape which is significantly different from the equilibrium 
one, as shown in Figure 7. We note that the curvature in front of the vesicle is higher than the one behind it. The reason is that the adhesion energy is higher in the front part, so that the curvature/adhesion balance allows a higher curvature (the vesicle looses curvature energy at the expense of a stronger adhesion).

\section{Stationary motion: an analytical solution}

The advantage offered by the simplistic picture of our model is the possibility to provide analytical results and thus to shed light on the physical processes that are involved in the problem of vesicle propulsion. It turns out that the equation of motion (34), if multiplied by $\partial^{2} \psi / \partial s^{2}$, possesses practically a first integral:

$$
\begin{aligned}
V \int_{0}^{L^{*}} \frac{\partial^{2} \psi}{\partial s^{2}} & \sin (\psi) \mathrm{d} s=\frac{\kappa}{\eta}\left(\left[\frac{1}{2}\left(\frac{\partial c}{\partial s}\right)^{2}\right]_{2}^{1}\right. \\
+ & \left.\frac{1}{8}\left[c^{4}\right]_{2}^{1}-\frac{\zeta}{2 \kappa}\left[c^{2}\right]_{2}^{1}-\frac{p}{\kappa}[c]_{2}^{1}\right) .
\end{aligned}
$$

Each r.h.s. term has an explicit form as a function of the contact curvatures (which are known), and of their first derivatives, which have only negligible contribution for swelled vesicle. The l.h.s. term can be evaluated for a vesicle shape close to a circle. The calculation will be given in the appendix and leads to

$$
\int \frac{\partial^{2} \psi}{\partial s^{2}} \sin (\psi) \mathrm{d} s=4 \pi^{2} \frac{L_{\mathrm{adh}}}{L^{2}} .
$$

Using the dynamical values for the contact curvature given by equation (22), we obtain an explicit expression for the velocity

$$
V=\frac{L^{2} \kappa}{(2 \pi)^{2} \eta L_{\mathrm{adh}}}\left(\frac{1}{2}\left[c^{4} / 4\right]_{2}^{1}-\frac{\zeta}{\kappa}\left[c^{2} / 2\right]_{2}^{1}-\frac{p}{\kappa}[c]_{2}^{1}\right) .
$$

In the simple case where $\Gamma=0$ (no dissipation associated with the substrate), expression (42) becomes explicit and provides a good agreement with numerical solution (see Fig. 8). The analytical expression for the velocity involves only known parameters, except $L_{\text {adh }}$ and $\zeta$. For the comparison between numerical and analytical results, we took their numerical values.

Another interesting limiting case concerns the small adhesion difference. Expansion of the numerator in equation (42) to leading order in $\delta w$ yields

$$
V \simeq \frac{\delta w}{\eta / A+\Gamma}, \quad A \equiv \frac{w}{\kappa} \frac{R^{2}}{L_{\mathrm{adh}}}\left[1-\frac{p}{w} \sqrt{\frac{\kappa}{2 w}}-\frac{\zeta}{w}\right],
$$

where $R=L / 2 \pi$. The influence of the two dissipation coefficients appears then clearly. It depends on the quantity $A$, proportional to the ratio $R / L_{\text {adh }}$. The bulk dissipation increases with the adhesion length, which seems to be a very robust result, as encountered in the model including

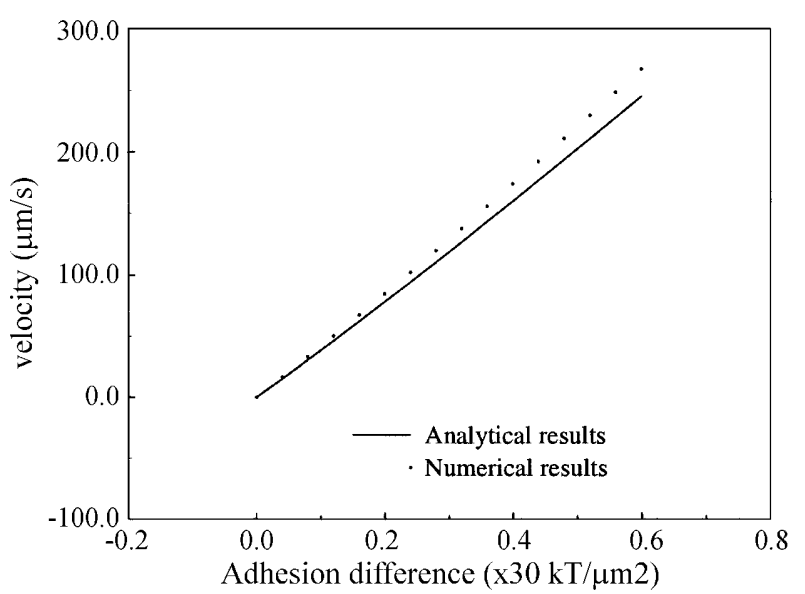

Fig. 8. Evolution of the vesicle velocity as a function of the adhesion difference.

hydrodynamics dissipation [10]. The local dissipation represented by $\Gamma$ does not depend on the adhesion length. Only the two contact points matter. Note also that the effective dissipation is $\eta / A+\Gamma$. The bulk dissipation $\eta / A$ and the contact one $\Gamma$ play the role of resistances (in an electric analogy) which would be mounted in series.

\section{Discussion and conclusion}

This paper has given a first extensive presentation of the problem of vesicle migration in haptotaxis. We have reduced as much as possible the complexity of the problem in order to gain some analytical approximate results. For that purpose we have neglected hydrodynamics which induce nonlocal interactions, and adopted a local model of the Rousse type. The full dynamical problem has been solved by adopting a powerful gauge-field invariant formulation. The dynamical code could account for the transient and the evolution towards a steady-state solution. In that context the introduction of an adhesion potential with a finite, albeit small, range has proven to be necessary in order to circumvent numerical instabilities related to the motion of the contact point. This code has the advantage of dealing with various problems not leading necessarily to permanent motions. For a stationary situation we could cast the problem into a standard boundary value one where the migration velocity appeared as an eigenvalue. This problem is akin to the nonlinear eigenvalue problem of Barenblat-Zeldovitch type. A counting argument showed us that the velocity should belong to a discrete set, only one solution has been identified; we speculate that the solution is unique. The problem could be systematically solved in a fully intrinsic representation of the contour. For a rather tense vesicle we have provided an analytical solution which is in a good agreement with the numerical one. We have identified the role played by the adhesion length in selecting the magnitude of the migration velocity even if no dissipation with the 
substrate is included. We have also shown that the bond breaking/restoring dissipation and the (effective) bulk one are additive in a way which is analogous to the problem of electrical resistances in series. Bulk dissipation dominates when the ratio of the bulk dissipation coefficient to the contact one exceeds a certain limit, which depends in an intricate manner on various parameters. For real situations, vesicles, and cells in general, are suspended in aqueous solutions. It is therefore highly important to include hydrodynamics. Moreover, the Lagrange multiplier $\zeta$ is a local quantity. We have recently given a brief account on these questions [10]. An extensive discussion will be presented in the near future.

\section{Appendix A. Derivation of equation (41)}

$$
D=\int_{0}^{L^{*}} \psi^{\prime \prime} \sin \psi \mathrm{d} s=-\int_{0}^{L^{*}}\left(\psi^{\prime}\right)^{2} \cos \psi \mathrm{d} s .
$$

We write $\psi=2 \pi s / L^{*}-\pi+\epsilon$, which implies to first order in $\epsilon^{\prime}$

$$
\begin{aligned}
D & =-\left(\frac{2 \pi}{L^{*}}\right)^{2} \int_{0}^{L^{*}} \cos \psi \mathrm{d} s-\frac{4 \pi}{L^{*}} \int_{0}^{L^{*}} \epsilon^{\prime} \cos \left(2 \pi s / L^{*}-\pi+\epsilon\right) \mathrm{d} s \\
& =-\left(\frac{2 \pi}{L^{*}}\right)^{2} L_{\mathrm{adh}}+\frac{4 \pi}{L^{*}} \int_{0}^{L^{*}} \epsilon^{\prime} \cos \left(2 \pi s / L^{*}+\epsilon\right) \mathrm{d} s .
\end{aligned}
$$

We then make use of the following relation:

$$
\frac{\mathrm{d}}{\mathrm{d} s}(\sin \psi)=-\left(\frac{2 \pi}{L^{*}}+\epsilon^{\prime}\right) \cos \left(2 \pi s / L^{*}+\epsilon\right) .
$$

The integral between 0 and $L^{*}$ of the l.h.s. term vanishes. We then obtain

$$
\int_{0}^{L^{*}} \epsilon^{\prime} \cos \left(2 \pi s / L^{*}+\epsilon\right) \mathrm{d} s=\frac{2 \pi}{L^{*}} \int_{0}^{L^{*}} \cos \psi \mathrm{d} s=\frac{2 \pi}{L^{*}} L_{\mathrm{adh}} .
$$

The sought-after relation has then the form

$$
D=\left(\frac{2 \pi}{L^{*}}\right)^{2} L_{\mathrm{adh}}
$$

\section{References}

1. W. Helfrich, Z. Naturforsch. C 28, 693 (1973).

2. Structure and Dynamics of Membranes, Handbook of Biological Physics, edited by R. Lipowsky, E. Sackmann (Elsevier, North-Holland, 1995).

3. F. Brochard, J.-F. Lennon, J. Phys. (France) 36, 1035 (1975).

4. J. Prost, R. Bruinsma, Europhys. Lett. 33, 321 (1996).

5. M. Kraus, W. Wintz, U. Seifert, R. Lipowsky, Phys. Rev. Lett. 77, 3685 (96).

6. I. Durand et al., Phys. Rev. E 56, 3776 (1997).

7. I. Cantat, C. Misbah, Phys. Rev. Lett. 83, 235 (1999).

8. I. Cantat, C. Misbah, in Transport and Structures, Their Competitive Roles in Biophysics and Chemistry, edited by S. Mueller, J. Parisi, W. Zimmermann (Springer, Berlin, 1999), Vol. 532, p. 93.

9. U. Seifert, Phys. Rev. Lett. 83, 876 (1999).

10. I. Cantat, C. Misbah, Phys. Rev. Lett. 83, 880 (1999).

11. J. Nardi, R. Bruinsma, E. Sackmann, Phys. Rev. Lett. 82, 5168 (1999)

12. S. B. Carter, Nature 213, 256 (1967).

13. A. S. G. Curtis, J. Embryol. Exp. Morphol. 22, 305 (1969).

14. As a leucocyte starts to crawl, part of its fluid cytoplasm turns rigid in a kind of sol-gel transition. The neutrophil extends a flat protrusion (leading lamella) that attaches to the underlying substrate, primarily through the action of membrane-adhesion proteins. The adhesion with the substrate provides a traction force that enables the cell to pull itself forward. The molecular motors provide a driving force to the actin filament that pushes the cell forward.

15. I. Cantat, Ph.D. thesis, Thèse de doctorat de l'Université Grenoble I, Grenoble, 1999.

16. Z. Csahók, C. Misbah, A. Valance, Physica D 128, 87 (1999).

17. U. Seifert, Phys. Rev. A 43, 6803 (1991).

18. R. Bruinsma, in Physics of Biomaterials, Fluctuations, Self Assembly and Evolution, edited by T. Riste, D. Sherrington, NATO ASI Ser. 332 (Kluwer, Dodrecht, 1996), p. 61.

19. P. de Gennes, Scaling Concepts in Polymer Physics (Cornell University Press, London, 1979).

20. The reason for locality is the following. For a true surface in $3 \mathrm{D}$ any motion on some scale $R$ induces a perturbation at least on that scale that couples different points on the surface. For a 1D object in 3D circulation of the liquid can wander around the line (or polymer-like geometry) without needing to affect distant points, leading thereby to local dynamics. 